\title{
PPP-RTK by Means of S-system Theory: Australian Network and User Demonstration
}

Odijk, D., Khodabandeh, A., Nadarajah, N., Choudhury, M., Zhang, B., Li, W. and Teunissen, P.J.G. 


\begin{abstract}
The development of an Australian PPP-RTK processing platform is an important component of a multi-GNSS enabled National Positioning Infrastructure. The PPP-RTK concept extends the precise point positioning (PPP) concept by providing single-receiver users with information enabling integer ambiguity resolution thereby reducing convergence times as compared to that of PPP. In this contribution we present and discuss the underlying principles of the PPP-RTK platform for both network and user. We demonstrate its GPS-based performance and provide an outlook for when multi-GNSS constellations, such as BDS, Galileo and QZSS, become fully operational.
\end{abstract}

Keywords: Global Navigation Satellite Systems (GNSS), PPP-RTK network and user, integer ambiguity resolution, common-clocks S-system, National Positioning Infrastructure

\title{
1. Introduction
}

Australia has adopted a "whole-of-nation" approach to implement a multi-GNSS enabled National Positioning Infrastructure (NPI; Australian Spatial Consortium, 2012). As a precursor to the NPI, the Australian Government is developing Analysis Centre Software, which is based on the PPP-RTK concept and aims at Australian-wide positioning with centimetre to decimetre accuracy (CRCSI, 2016a). The prototype (Matlab) PPP-RTK Network and User Platform software developed at Curtin University forms one of the key inputs to the Analysis Centre Software (CRCSI, 2016bc). Based on the current status of this first Australian-made PPP-RTK Platform, this contribution presents an overview of the underlying principles of the software and a demonstration of its performance.

The PPP-RTK method, first described by Wübbena et al. (2005), is based on the Precise Point Positioning (PPP) method introduced by Zumberge et al. (1997). In contrast to the PPP method, the PPP-RTK method utilizes information to resolve the carrier-phase ambiguities to integers, similar to the Real-Time Kinematic (RTK) relative positioning method, but then for a single GNSS receiver. The benefit of integer ambiguity resolution allows the PPP-RTK user to obtain faster convergence to high (centimetre level) positioning accuracy than what is possible with standard PPP.

Several PPP-RTK implementations exist, see Teunissen and Khodabandeh (2015) for an overview and comparison. The method underlying Curtin's PPP-RTK Platform software is based on the undifferenced and uncombined GNSS observation equations (Odijk et al., 2016), which means that at the observation level no combinations are formed, such as, for example, single or double differences, or (ionosphere-free) combinations of observations at different frequencies. The undifferenced approach is most flexible, as it allows for dynamic constraints on all parameters, thus including parameters that would be eliminated in a differencing approach, which is beneficial for strengthening the observation model. Another advantage of the undifferenced approach is that a multi-GNSS integration, with multiple GNSSs having parameters in common, can be modelled in a rigorous way (Khodabandeh and Teunissen, 2016). Also, as ionospheric parameters are not eliminated but estimated in the undifferenced and uncombined approach, these can be used as input to GNSS-based ionospheric modelling studies. 
Curtin's PPP-RTK Platform software consists of a network and a user component, see Figure 1. These two components are developed as separate platforms, referred to as the Network Platform and the User Platform (CRCSI, 2016bc). Input for the Network Platform are dual-frequency GPS phase and code data of receivers of a Continuously Operating Reference Station (CORS) network, for example an Australian-wide network. Together with (external) information about the satellite orbits and station positions, these GPS data are processed by the Network Platform to produce correction information (i.e. satellite clocks and satellite phase biases for both frequencies) that allows PPP-RTK for a single-receiver GPS user who applies this information for correcting his or her phase and code data. These corrected data form, together with a rough, approximate user position, as well as precise orbit information, input for the User Platform that outputs PPP-RTK positions for the user receiver.

The structure of this contribution is as follows. Section 2 presents the underlying model and the estimable parameters of Curtin's PPP-RTK Network and User Platforms. This is followed by an overview of the functionalities of the respective platforms in Section 3. Results based on dualfrequency GPS data tracked by an Australian-wide GPS CORS network are presented and discussed in Section 4. Section 5 provides then an outlook and initial results of further improvements that can be expected when incorporating ionospheric corrections and when integrating multiple GNSSs. Finally, the conclusions are presented in Section 6.

\section{Estimable parameters of Curtin's PPP-RTK Platforms}

\section{GNSS phase and code observation equations}

Basis for the PPP-RTK Network and User Platforms are the nonlinear GNSS observation equations for phase and code (e.g., Teunissen and Kleusberg, 1998):

$$
\begin{aligned}
& \phi_{r, j}^{s}=\rho_{r}^{s}+\left(d t_{r}-d t^{s}\right)+\tau_{r}^{s}-\mu_{j} l_{r}^{s}+\lambda_{j}\left(\delta_{r, j}-\delta_{, j}^{s}+z_{r, j}^{s}\right)+\varepsilon_{r, j}^{s} \\
& p_{r, j}^{s}=\rho_{r}^{s}+\left(d t_{r}-d t^{s}\right)+\tau_{r}^{s}+\mu_{j} l_{r}^{s}+\left(d_{r, j}-d_{, j}^{s}\right) \quad+e_{r, j}^{s}
\end{aligned}
$$

The above variables have a receiver index $r=1, \ldots, n$, with $n$ the number of receivers, a frequency index $j=1,2$, and a satellite index $s=1, \ldots, m$, with $m$ the number of satellites. Apart from the ambiguities that are constant during a satellite pass (provided no cycle-slips occur), all variables are time dependent. For simplicity we do not include a time index in their notation, but in the software platforms this is properly taken into account (including dynamic models).

The variables in (1) have the following meaning:

$\begin{array}{ll}\phi_{r, j}^{s}: & \text { phase observable } \\ p_{r, j}^{s}: & \text { code observable } \\ \rho_{r}^{s}: & \text { receiver-satellite range } \\ d t_{r}: & \text { receiver clock bias } \\ d t^{s}: & \text { satellite clock bias } \\ \tau_{r}^{s}: & \text { tropospheric bias } \\ \iota_{r}^{s}: & \text { ionospheric bias } \\ \mu_{j}: & \text { ionospheric coefficient } \\ \lambda_{j}: & \text { wavelength } \\ \delta_{r, j}: & \text { receiver phase bias }\end{array}$


$\delta_{, j}^{S}: \quad$ satellite phase bias

$z_{r, j}^{S}: \quad$ phase ambiguity

$d_{r, j}: \quad$ receiver code bias

$d_{, j}^{S}: \quad$ satellite code bias

$\varepsilon_{r, j}^{S}: \quad$ phase observation noise

$e_{r, j}^{S}: \quad$ code observation noise

All variables in (1) are expressed in meters, except the phase-related parameters (i.e. receiver and satellite biases, plus ambiguities), that are expressed in cycles. The term 'bias' is referred to as any combination of the instrumental delays associated with the receivers and satellites. The ionospheric coefficient is defined as the squared ratio of wavelengths: $\mu_{j}=\left(\lambda_{j} / \lambda_{1}\right)^{2}$. For dual-frequency GPS, $\mu_{1}=1$ and $\mu_{2}=\left(\frac{77}{60}\right)^{2}$. The ionospheric delay parameters therefore relate to the first (i.e. L1) frequency.

In case of a PPP-RTK network the receiver-satellite ranges $\rho_{r}^{S}$ are known, as the satellite positions are calculated from precise orbits from the International GNSS Service (IGS; Dow et al., 2009) and the receiver positions are based on precise a-priori values. These receiver-satellite ranges are therefore subtracted from the observations.

The observation equations (1) also apply to a PPP-RTK user, with the exception that the user operates a single receiver $(n=1)$, denoting the user index as $r=u$. Moreover, the user's observation equations need to be linearized with respect to the receiver position, as this is unknown. Another difference with the network is that the user applies corrections for satellite clocks and phase biases from the network (see Figure 1) and these are subtracted from the user's observations. Considering the ionospheric delays, there are two options: (i) the user estimates unknown parameters for the ionospheric delays ("ionosphere-float PPP-RTK user"), or (ii) the user corrects the observations for the ionospheric delays based on corrections from the network ("ionospherecorrected PPP-RTK user").

\section{PPP-RTK Network Platform parameters}

The parameters in (1) are not unbiasedly estimable, because of rank deficiencies in the system of observation equations. By means of S-system theory these rank deficiencies can be identified and eliminated (Odijk et al., 2016). Other PPP-RTK models such as those given in e.g., Laurichesse and Mercier (2007), Collins (2008) and Ge et al. (2008) were also shown to follow as an application of Ssystem theory (Teunissen and Khodabandeh 2015). One choice of S-basis leads to the Common Clocks S-system, for which the estimable network parameters are, together with their interpretation, given in Table 1. The parameters that are fixed as S-basis are also given in the table. This S-basis choice is motivated by the satellite clocks that are, similar to the IGS clocks, estimable as ionosphere-free clocks (Kouba and Heroux, 2001).

In Table 1 the ionosphere-free (IF) and geometry-free (GF) combinations of receiver and satellite code biases frequently appear. For GPS the ionosphere-free coefficients are $\frac{\mu_{2}}{\mu_{2}-\mu_{1}} \approx 2.55$ and $\frac{\mu_{1}}{\mu_{2}-\mu_{1}} \approx 1.55$, whereas the geometry-free coefficient equals $\frac{1}{\mu_{2}-\mu_{1}} \approx 1.55$. In this context, note that $d_{, 1}-d_{, 2}$ is the Differential Code Bias (DCB; either for receiver or satellite), so $d_{, G F}$ is a scaled DCB. The 
ionosphere-free and geometry-free code biases are related to their undifferenced counterparts as $d_{, I F}+\mu_{j} d_{, G F}=d_{, j}$, for $j=1,2$ (Teunissen and Khodabandeh, 2015).

The tropospheric delays are decomposed as $\tau_{r}^{S}=\tau_{r, 0}^{S}+\mathrm{m}_{r}^{S} \tau_{r}$, with $\tau_{r, 0}^{S}$ the a-priori tropospheric delay (coming from a troposphere model), $\mathfrak{m}_{r}^{s}$ the tropospheric mapping function and $\tau_{r}$ the (residual) zenith tropospheric delay (ZTD), which is estimable per receiver, see Table 1.

Apart from the ZTDs, the estimable parameters are all biased, as they are lumped to other parameters. The receiver and satellite clocks are estimable clocks lumped with the ionosphere-free combination of code biases, making them 'ionosphere-free' clocks. The ionospheric delays are estimable biased with the geometry-free combination of code biases. Apart from the ZTDs and ionospheric delays, all other estimable parameters are furthermore relative with respect to receiver 1, which is the network's reference receiver. In addition to this reference receiver, the ambiguities of a reference satellite (i.e. satellite 1) show up in the estimable receiver phase biases and ambiguities. Due to this reference satellite however, the estimable ambiguities are double differenced and thus integer.

For simplicity, the interpretation of the network parameters in Table 1 is based on the assumption that all receivers in the network observe all satellites. In Curtin's PPP-RTK Network Platform software this strict assumption does however not apply. By using the minimum spanning tree concept (De Jonge, 1998), widely separated or even globally distributed receivers can be processed. The estimable ambiguities are "double-differenced like" and thus integer, i.e. not necessarily combination of four undifferenced ambiguities as in Table 1, but always linear combinations of doubledifferenced ambiguities.

\section{Ionosphere-float PPP-RTK User Platform parameters}

The network parameters that enable PPP-RTK for the user are the satellite-related parameters from Table 1:

- $\quad$ satellite clocks (denoted as $d \tilde{t}^{s}$ );

- $\quad$ satellite phase biases for each frequency (denoted as $\tilde{\delta}_{, j}^{s}, j=1,2$ ).

The estimability and interpretation of the ionosphere-float PPP-RTK user parameters is automatically obtained from their network counterparts in Table 1 (by inserting $r=u$ for the user), where it is, like the network, assumed that the user operates on two frequencies. There is one difference, namely that the user's position (denoted as $x_{u}$ ) is estimated as well. The estimable user's phase ambiguities, are, like the network ambiguities, double-differenced and thus integer. The user's ambiguities are estimable relative to the network's reference receiver, although the user does not need to know which network receiver this actually is. On the reference satellite, the user is free to choose this, so it does not have to be the same reference satellite as the network. The choice of this reference satellite implies that the user's estimable receiver phase bias is -like the estimable ambiguity-also relative to this reference satellite.

\section{Ionosphere-corrected PPP-RTK User Platform parameters}


It is known that the presence of the unknown ionospheric delay parameters has an adverse effect on the convergence time for the user, as the ionosphere-float PPP-RTK model is rather weak in terms of successful integer ambiguity resolution. Faster convergence times are expected if the user would correct for these ionospheric delays. In fact, Curtin's PPP-RTK Network Platform estimates (biased) ionospheric delays for every receiver in the network (see Table 1), but in order for the user to include ionospheric corrections, these network ionospheric delays need to be predicted to the location of the user, e.g. by means of spatial interpolation or an ionosphere model.

Assume that the PPP-RTK user applies the following ionospheric corrections from the network:

$\tilde{\iota}_{\text {net }}^{S}=\iota_{u}^{S}+d_{\text {net,GF}}-d_{, G F}^{S}$

These ionospheric corrections are biased by (scaled) DCBs, where the network receiver-dependent part, denoted as $d_{n e t, G F}$, is a certain function of the DCBs of the receivers in the network.

Because of the ionospheric corrections, also single-frequency PPP-RTK is possible. Therefore we distinguish between an ionosphere-corrected dual-frequency and a single-frequency PPP-RTK user.

\section{Ionosphere-corrected dual-frequency PPP-RTK user}

Table 2 presents the estimable PPP-RTK user parameters, as well as the chosen S-basis, in the presence of ionospheric corrections for a dual-frequency user.

The estimability and interpretation of the position, ZTD and ambiguities is identical to the ionosphere-float PPP-RTK user, but the interpretation of the user's phase bias parameter has changed due to the ionospheric corrections. Basically, the scaled user DCB $d_{u, G F}$ is replaced by its network counterpart $d_{n e t, G F}$. This has however no consequence for the estimability of the user's phase bias parameter. An important consequence of incorporating ionospheric corrections is that a receiver code bias parameter becomes estimable. A dual-frequency, ionosphere-corrected PPP-RTK user thus estimates the difference of DCBs between user and network, i.e. $d_{u, G F}-d_{n e t, G F}$ as additional parameter. Note that only one parameter is estimated for both frequencies, but this parameter is multiplied by the frequency-dependent ionospheric coefficient $\mu_{j}$.

\section{Ionosphere-corrected single-frequency PPP-RTK user}

In the single-frequency ionosphere-corrected PPP-RTK case there are no receiver code bias parameters estimable, which is a consequence of a rank deficiency between the user's clock and single-frequency phase and code bias parameters. As consequence, the interpretation of the estimable clock and phase bias parameters in the single-frequency case is different from those in the dualfrequency case, see Table 3, which presents the estimable parameters as well as S-basis for a singlefrequency (denoted using frequency 1), ionosphere-corrected PPP-RTK user.

\section{A-priori corrections}


Besides a correction for the tropospheric delays as mentioned earlier, both PPP-RTK network and user data need to apply a series of so-called a-priori corrections. An overview of these corrections, including their magnitude and source, is presented in Table 4.

We remark that Curtin's PPP-RTK Network Platform aligns its estimable satellite clocks for GPS to the P1 and P2 code observables, which is similar to the IGS procedure. This means that for receivers employing the $\mathrm{C} 1$ (C/A) code observable, the P1-C1 DCB corrections need to be applied.

\section{Functionalities of Curtin's PPP-RTK Platforms}

This section presents an overview of the functionalities of Curtin's PPP-RTK Network and User Platforms. Figure 2 depicts flowcharts of both platforms. As the designs of both platforms are similar, their functionalities are described together. Both software platforms can be divided into three parts:

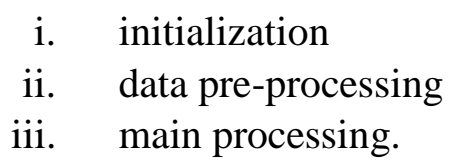

In the initialization part the so-called steering file is read in, together with the input files for the orbits and antenna offsets. In case of the Network Platform also the file with station positions is read in. The steering file is the file that 'steers' the software as it contains, among others, the settings for the stochastic model of the observations and the dynamic model of the estimable parameters, as well as the 'treatment' of the ionosphere in case of the User Platform.

After the initialization stage, the data pre-processing starts, consisting of the following four blocks for both Network and User Platforms (see Figure 2):

1. Epoch-wise reading (and epoch matching): This block is to read, in an epoch-by-epoch way, the observation data, which are provided in RINEX format (either version 2 or 3, see IGS and RTCM-SC104, 2015), as well as to read the receiver PCO/PCVs corrections from the IGS ANTEX file. In case of the Network Platform the observation epochs among all receivers in the network are synchronized. In case of the User Platform, the file with PPP-RTK corrections from the Network Platform is read in and the epochs of these corrections are synchronized with the epochs of the single-receiver observation file.

2. Single-channel DIA: This block implements the geometry-free, single-channel DetectionIdentification-Adaptation (DIA) algorithm (Teunissen and De Bakker, 2013). This initial DIA procedure is performed on a channel-by-channel basis (i.e. per receiver-satellite combination), without the need for external information (such as satellite orbits or clocks). The purpose of the single-channel procedure is, as a first quality control procedure, to identity and remove large model errors from the data.

3. Orbit interpolation (and SPP): This block is to compute, per receiver, the satellite orbit positions at the time of signal transmission. In case of the User Platform, also a code-based Single Point Positioning (SPP) is performed as to provide approximate values for the user's position if these are unavailable. In this block also the observations are removed that are 
below the cut-off angle as specified in the steering file. Moreover, relativity effects and troposphere corrections are computed.

4. A-priori corrections: In this block the a-priori corrections as listed in are applied to the phase and code observations. As a result, 'observed-minus-computed' observations are obtained.

The following two blocks together form the main processing part:

5. Float solution: This block is the core function of both the Network and User Platforms and consists of the following two sub-blocks:

- Generalized filter: Like a Kalman filter, this block contains a time update step to predict the state vector from the previous epoch to the current epoch based on the dynamic model settings. The difference with the standard Kalman filter is that Curtin's PPP-RTK platforms make use of the so-called Generalized filter, in which it is not required that all parameters have a dynamic model, but only a subset of these parameters, or in the extreme case, none of the parameters. For example, in case of an unstable receiver clock one can choose to estimate them without dynamic model, whereas at the same time, the ambiguities should be modelled as time-constant parameters. After the time update for the parameters for which a dynamic model is specified (through the steering file), the measurement update step combines the predicted state vector parameters with the observations of the current epoch and a 'filtered' state vector is obtained. The Generalized filter is initialized by performing a standard least-squares estimation based on the observations of the first epoch.

- Main DIA: In parallel to the above filtering step a Detection-Identification-Adaptation procedure is executed to warrant the filter results from the unwanted effects of potential model errors, such as phase slips and code outliers. In contrast to singlechannel DIA, this 'main' DIA procedure is based on the full observation model (Teunissen, 1990).

6. Fixed solution: The Generalized filter outputs the so-called 'float' solution, which means that the ambiguities are estimated as real-valued parameters. The float solution is used in this block to obtain the solution based on resolved integer ambiguities, the so-called 'fixed' solution:

- Integer Ambiguity Resolution (IAR): This block is to perform integer carrier-phase ambiguity resolution based on the float ambiguity solution, which is input to the LAMBDA method (Teunissen, 1995). Instead of resolving the full ambiguity vector, the software user can opt to resolve a subset of the ambiguities, i.e. partial ambiguity resolution (PAR; Teunissen et al., 1999). Given a user-defined minimum success-rate, the maximum number of float ambiguities having success-rate higher than the stated minimum value are to be fixed to their integers. By having PAR as an option, one avoids fixing ambiguities of poor precision to ensure the minimum success-rate. Note that IAR is implemented as an option in both software platforms; the software user can either turn this on or off. 
- Least Squares: After the integer ambiguities have been estimated, all other parameters are updated by means of a standard least-squares adjustment based on the fixed ambiguities and the fixed solution is obtained.

Output of the Network Platform are, among others, the PPP-RTK network products (cf. Figure 1), i.e. the parameters that enable PPP-RTK for a single receiver. In Figure 2 these are written to files and these files form one of the inputs for Curtin's PPP-RTK User Platform software.

\section{PPP-RTK results based on an Australian-wide GPS network}

With the use of dual-frequency GPS data, we tested the feasibility of our PPP-RTK methodology, at both the network and the user sides. In case of the network, 24-hour data of 24 Australian CORS receivers (managed by Geoscience Australia) were collected on 8 February 2015 with 30 second sampling interval. Their sparse geographic distribution is shown in Figure 3. Station 1 (ALBY; Albany in Western Australia) is selected as the network's reference receiver.

\section{PPP-RTK Network Results}

For the network processing using Curtin's PPP-RTK Network Platform the satellite positions were obtained using precise IGS orbits, whereas precise positions of all stations were extracted from IGS SINEX (Solution Independent Exchange format) files. In addition, as to align the estimable satellite clocks to P1 and P2 observables, P1-C1 satellite DCBs using the monthly products of the Centre for Orbit Determination in Europe (CODE) were applied to stations tracking C1 code data. The cut-off angle of the observations was set to 10 degrees. All phase and code observations were weighted according to the sine of their elevation, with an undifferenced standard deviation in zenith of $3 \mathrm{~mm}$ for the phase data and $30 \mathrm{~cm}$ for the code data. Concerning the dynamic models of the parameters, the receiver and satellite phase biases, as well as ambiguities, were assumed to be time constant, whereas the receiver and satellite clocks, as well as the ionospheric delays, were assumed to be unlinked in time. For the ZTDs a random-walk stochastic process was assumed with a process noise of $1 \mathrm{~mm}$ per $\sqrt{30}$ seconds. Concerning integer ambiguity resolution, the software was set to Partial Ambiguity Resolution, with a minimum success rate of 0.999 .

Figure 4 contains three panels. The upper panel summarizes, for each GPS satellite, the time period(s) during which that particular satellite is in common view of at least two receivers. As a complement to the upper panel, the middle panel depicts the number of satellites as a function of time. As shown, the number of satellites that are visible above 10 degrees varies from 9 to 15 . The bottom panel shows the time-series of Local Overall Model (LOM) test statistics (Teunissen, 1990), along with their critical values. These LOM test values are obtained in the Detection step, the first step of the Main DIA procedure. During the day all LOM values are found to be less than their critical values, indicating the validity of the network model used.

Figure 5 shows the satellite clock estimates $\left(d \tilde{t}^{s}\right)$ (see Table 1), together with their formal standard deviations, for passes of 4 arbitrarily satellites (i.e. PRN 7, 18, 23 and 29). It can be seen that for each satellite shown its clock estimates behave almost linearly as a function of time, which can be expected for the behaviour of the GPS satellite clocks. At the same time, it usually takes about 2 hours to have satellite clock estimates with formal standard deviations lower than $2 \mathrm{~cm}$. 
Referring to the same 4 satellites involved in Figure 5, Figure 6 shows the satellite phase bias estimates for the L1 frequency $\left(\tilde{\delta}_{, 1}^{s}\right)$ (see Table 1), plus their formal standard deviations. Instead of showing the satellite phase biases for L2 as well, Figure 7 depicts their difference, which is known as the wide-lane satellite phase bias $\left(\tilde{\delta}_{, W L}^{s}=\tilde{\delta}_{, 1}^{s}-\tilde{\delta}_{, 2}^{s}\right)$. The reason for showing these wide lanes is that they are more precise than their uncombined counterparts. Apart from the jumps, it can be seen that both L1 and wide-lane satellite phase biases are quite stable during a satellite's pass. The formal precision of the L1 satellite phase biases reaches a stable level of about 0.15 cycle after 3 hours, whereas a low standard deviation of 0.05 cycle is already reached after 1 hour for the wide-lane satellite phase biases. The jumps that are visible in both L1 and wide-lane satellite phase bias estimates are caused by a change in reference ambiguity $z_{1, j}^{S}$ (see Table 1 ).

Although not provided to the PPP-RTK users, in Figure 8 the ionospheric delay estimates associated with the network's reference receiver ALBY $\left(\tilde{l}_{1}^{S}\right)$ and the 4 aforementioned satellites are plotted. Note that, for each satellite, the ionospheric delay estimates may take on negative values. This is because the slant ionospheric delay estimates are biased by the (scaled) receiver and the satellite DCBs (see Table 1). These estimates, whose formal standard deviations are smaller than $5 \mathrm{~cm}$ after convergence (around 2 hours), may act as basic inputs for GPS-based ionosphere modelling.

\section{PPP-RTK User Results}

The network-derived satellite clock and satellite phase bias products were in a next step applied to correct the phase and code data of about 300 single-receiver dual-frequency GPS users, distributed over entire Australia. The associated settings for Curtin's PPP-RTK User Platform were identical to the PPP-RTK network processing (Table 1), except that we did the processing for each receiver twice, as to investigate different settings in the dynamic model of the receiver position:

- $\quad$ static positioning, i.e. keeping the receiver position constant in time;

- kinematic positioning, i.e. treating the receiver position completely unlinked in time.

Both static and kinematic positioning were conducted without and with integer ambiguity resolution, i.e. ambiguity-float vs. ambiguity-fixed.

To get an initial impression of the role played by single-receiver ambiguity fixing, first consider the kinematic positioning performance of one of the PPP-RTK users, which is receiver located in Orbost in Victoria (ORBO). The time-series of the fixed user position with respect to its ground-truth (in East-North-Up) is shown in Figure 9. To see the improvement brought by ambiguity resolution, the fixed results are compared with their float counterparts in Figure 10, for the first 8 hours. Note that the difference between the fixed and float results is significant at the beginning of the time-series, while this difference gets smaller over time. This is what one would expect as the time-constant float ambiguities get more precise over time, leading the phase observations to govern the positioning solutions. The role of successful single-receiver ambiguity-fixing is therefore to reduce the positioning convergence time. If one defines the convergence time as the minimum observational time required to achieve an absolute positioning error less than $10 \mathrm{~cm}$, the convergence times of the East, North and Up components are 24, 24 and 31 minutes, respectively, in the ambiguity-fixed case. The corresponding convergence times of their float counterparts are 105, 26 and 137 minutes, respectively. Mainly the East and Up components significantly benefit from ambiguity resolution. The term 'absolute' refers to the difference with respect to a ground-truth value. 
Now the question is whether the aforementioned convergence times of this single PPP-RTK user station are 'representative' or not. To address this, we recall that the GNSS data, like any other measured data, are accompanied by an amount of uncertainty. The positioning convergence times, obtained by the GNSS data, are therefore of a random nature. This means that a large number of samples are required to infer their distributions. For this purpose we processed about 300 singlereceiver user stations and for every observational epoch, about 300 horizontal radial absolute errors of the users' positions with respect to their ground-truth are computed.

Figure 11 shows the resulting convergence curves for static positioning. The $50 \%, 75 \%$ and $90 \%$ percentiles of these values are plotted as function of time. In case of ambiguity-float, the results show that the horizontal radial absolute error of $50 \%$ of the stations gets less than $10 \mathrm{~cm}$ after 25 minutes, whereas that of $90 \%$ of the stations attains $10 \mathrm{~cm}$ after 60 minutes. For the case of ambiguity-fixing, we note the favourable impact of integer ambiguity resolution through the much steeper decrease of the convergence curves. After ambiguity-fixing, $50 \%$ of the positioning errors already get less than $10 \mathrm{~cm}$ within 20 minutes, while $90 \%$ of them attain $10 \mathrm{~cm}$ after 42 minutes. Let us now focus our attention on smaller positioning errors of less than $2 \mathrm{~cm}$. Over 120 minutes (2 hours) is required for $75 \%$ of the float errors to become less than $2 \mathrm{~cm}$, while $90 \%$ of which need more than 3 hours to attain $2 \mathrm{~cm}$. In case of the fixed curves however, after 30 minutes $75 \%$ of the errors become less than $2 \mathrm{~cm}$. After just 45 minutes, $90 \%$ of the ambiguity-fixed results become less than $2 \mathrm{~cm}$.

Figure 12 shows the convergence curves for kinematic positioning, which are plotted for the same receivers as the static positioning. Now the receiver positions are assumed to be completely unlinked in time, resulting in a weaker model than the static positioning model. This weaker model has its effect on the convergence times. In the ambiguity-float case more than 180 minutes ( 3 hours) is required in order for $50 \%$ of the absolute positioning error become less than $2 \mathrm{~cm}$. As comparison, in the static case about 90 minutes are required. Fortunately, ambiguity resolution also improves the convergence times in the kinematic case. It can be clearly observed from Figure 12 that the $90 \%$ percentile steeply drops from $20 \mathrm{~cm}$ to almost $2 \mathrm{~cm}$ after 50 minutes, i.e. achieving a 10 times improvement.

\section{PPP-RTK: Outlook}

The results of the previous section are based on ionosphere-unknown, dual-frequency GPS-only data. In this section we discuss two developments that will further improve this PPP-RTK performance. The first is multi-frequency, multi-GNSS PPP-RTK, which is driven by the emerging global and regional navigation constellations that are currently under development, and the second is ionosphere-corrected PPP-RTK, which is driven by the development of an ionospheric model for the Australian continent (CRCSI 2016d). In the following we discuss their estimability impact and illustrate the performance improvements that can be expected.

\section{Multi-frequency, multi-GNSS PPP-RTK}

Both Curtin's PPP-RTK Network and User Platforms currently (mid-2016) support legacy (dualfrequency) GPS. The ultimate goal for the software development is multi-frequency, multi-GNSS, 
supporting the following global constellations: modernized GPS, modernized (CDMA-based) GLONASS, BDS (BeiDou) and Galileo.

The new constellations and also modernized GPS will transmit signals on more than two frequencies and this has as consequence that in addition to phase bias parameters for each of the new frequencies also code bias parameters become estimable (Odijk et al., 2016). These code bias parameters, for both receiver and satellite, however only apply from the third frequency onwards, see Table 5. This implies that the network has to estimate and transmit these satellite code biases as additional product to triple- or higher-frequency PPP-RTK users. Remark that these additional code biases should also be applied in case of triple- or higher frequency standard PPP, see also Elsobeiey (2015). The multifrequency PPP-RTK user has to parameterize additional receiver code biases as well. For the ionosphere-float PPP-RTK user the interpretation of this receiver code bias parameter follows by inserting $r=u$ in the expression in Table 5.

In this context, an equivalent interpretation for the part of the code bias between round brackets in Table 5 can be given as:

$d_{, j}-\left[d_{, I F}+\mu_{j} d_{G F}\right]=-\left[\left(d_{, 1}-d_{, j}\right)-\frac{\mu_{j}-\mu_{1}}{\mu_{2}-\mu_{1}}\left(d_{, 1}-d_{, 2}\right)\right], \quad j \geq 3$

In this expression we omitted receiver or satellite indices, as this expression applies to both. Apart from the sign, the code bias, can thus also be interpreted as a combination of the "modernized" DCB $d_{, 1}-d_{, j}$ and the "legacy" DCB $d_{, 1}-d_{, 2}$.

The ionosphere-corrected PPP-RTK user already has to estimate a receiver code bias parameter, although not in the single-frequency case, but in the dual-frequency case, see Table 2 . In a triple- or higher-frequency case the interpretation of the estimable receiver code bias parameter can be given as:

$\tilde{d}_{u, j}=\left(d_{u, j}-\left[d_{u, I F}+\mu_{j} d_{n e t, G F}\right]\right)-\left(d_{1, j}-\left[d_{1, I F}+\mu_{j} d_{1, G F}\right]\right), \quad j \geq 3$

In case $j=1,2$ this expression reduces to the estimable receiver code bias $\tilde{d}_{u, j}=\mu_{j}\left(d_{u, G F}-\right.$ $\left.d_{n e t, G F}\right)$ for the dual-frequency ionosphere-corrected case (see Table 2).

Besides the additional PPP-RTK parameters due to extra frequencies, another aspect of multi-GNSS that will have an effect on PPP-RTK is that the mentioned constellations transmit signals on identical frequencies, for example the QZSS frequencies that are identical to those of GPS. Both PPP-RTK Network and User Platforms should make use of this, as knowledge of the inter-system biases (ISBs, i.e. the difference between hardware biases of different constellations but on identical frequencies) strengthens the multi-GNSS observation models. Khodabandeh and Teunissen (2016) present an approach to make use of an "ISB look-up table" to strengthen multi-GNSS PPP-RTK for the user.

We will now present results for some multi-GNSS PPP-RTK scenarios, based on multi-frequency data of GPS, Galileo, BDS and QZSS. PPP-RTK products were estimated from a single station instead of a network, using the multi-GNSS receiver in Fitzroy Crossing, Western Australia (FROY). The multi-GNSS receiver located in Wallal in Western Australia (WLAL), at a distance of almost $600 \mathrm{~km}$ from FROY, acts as PPP-RTK user. The PPP-RTK positions of WLAL were estimated in kinematic mode (i.e. unlinked in time) and compared to the ground-truth position, for 12 hours (10:50-22:50 GPST) of 8 June 2016. Precise GBM orbits produced by the German Research Centre for Geosciences (GFZ; Guo et al., 2016) were used to compute the positions of the GPS, Galileo, 
BDS and QZSS satellites. The number of satellites that are tracked above 10 degrees cut-off elevation are for GPS between 6 and 11, for Galileo between 1 and 5, for BDS between 9 and 12 and 1 QZSS satellite. On average more BDS satellites than GPS satellites are tracked for this location in Western Australia. Table 6 gives the frequencies of the different constellations that are used in the PPP-RTK processing. Note that both Galileo and QZSS transmit on a third frequency, for which satellite code biases (see Table 5) need to be applied for PPP-RTK. Although GPS also transmit signals on a third frequency, these signals are not used here as they are not transmitted by the whole constellation, but only by the new GPS satellites. At the time of data collection, these 8 new satellites are PRNs 1, 3, 6, 9, 24, 25, 27 and 30.

Figure 13 illustrates the improvements that one can expect in the ambiguity-fixed convergence times when adding constellations to the dual-frequency GPS. These improvements are only based on the currently operational satellites and therefore not yet on the fully operational constellations. Even further improvements can therefore be expected when more satellites of these systems get included as well as when also including IRNSS and GLONASS (note: the Indian IRNSS was excluded as no precise orbits were yet available, while GLONASS was excluded due to the current lack of CDMA data). It follows from Figure 13 that for a positioning error of $5 \mathrm{~cm}$ horizontally and $10 \mathrm{~cm}$ vertically, the convergence time of GPS standalone is 53 minutes for both East and Up, whilst 17 minutes for North. However, when BDS is added to GPS, the convergence times improve significantly from 53 minutes to 11 minutes for all components. This improvement is due to the relatively large number of BDS satellites that can be tracked. The triple-constellation combination of GPS, BDS and Galileo, with convergence times of 10 minutes, gives a further improvement, but smaller than when adding BDS to GPS. This is due to the fewer satellites of Galileo that are currently available. Adding QZSS as a fourth constellation but with only one satellite, will at first sight not improve the convergence times of GPS+BDS+Galileo. However, by making use of the property that QZSS transmits on identical L1 and L2 frequencies as GPS for which the inter-system biases can be assumed known, the single QZSS satellite further reduces the convergence times, with 1 minute compared to GPS+BDS+Galileo. Figure 14 depicts the time-series of the ambiguity-float and ambiguity-fixed positioning errors for the quadruple-constellation combination for the first 5 hours (i.e. 10:50-15:50 GPST). While the horizontal components are improved by ambiguity-fixing, little improvement is observed for the Up component.

\section{PPP-RTK incorporating ionospheric corrections}

Next to multi-GNSS integration, another factor that will benefit the PPP-RTK user-convergence is the availability of precise ionospheric corrections. To get an impression of the contribution of ionospheric corrections to the convergence time of GPS-only PPP-RTK, we made use of the CORS station ionospheric delay estimates as outputted by the Network platform. By means of linear interpolation these estimates were used to predict the ionospheric corrections for a user located at an average distance of $100 \mathrm{~km}$ from the network stations. Inclusion of these ionospheric corrections in the PPP-RTK corrections showed that the time required reduced to only a few minutes for the ambiguity-fixed kinematic user-positioning error to be below $5 \mathrm{~cm}$ horizontally and $10 \mathrm{~cm}$ vertically (compare with the GPS-only results of Figure 13). This clearly demonstrates the key role the ionospheric corrections can play in reducing the positioning convergence times. As this result is GPS-only based and obtained without the use of an ionospheric model, one can expect even further improvements in case a precise ionospheric model becomes available. Such a high-resolution 
ionosphere model for the Australian continent is currently under development (CRCSI, 2016d). And one can expect that multi-GNSS will also here have a beneficial effect on the quality of the ionosphere model, as the ionosphere gets intersected by many more lines-of-sight than is the case with GPS alone.

\section{Conclusions}

The development of an Australian PPP-RTK processing platform is an important component of a multi-GNSS enabled National Positioning Infrastructure. In this contribution we presented the underlying principles of the PPP-RTK platform, for both network and user, demonstrated its performance and provided an outlook for when the multi-GNSS constellations become fully operational.

Both the PPP-RTK Network and User platforms are developed on the basis of undifferenced and uncombined GNSS observation models because of the flexibility it offers. They employ recursive estimation (implemented as a Generalized filter), with in parallel a recursive quality control procedure. As all parameters are maintained in the model, it allows for dynamic constraints on the parameters that have a strengthening effect on the models. Moreover, it allows expansion of the undifferenced models to include multi-frequency, multi-GNSS data. The interpretation of the platforms estimable parameters was presented in the framework of the common-clocks approach for both the ionosphere-float and ionosphere-corrected models.

The performance of both platforms was demonstrated for a network of 24 CORS stations sparsely distributed over Australia and tracking dual-frequency GPS data. PPP-RTK products (satellite clocks and satellite phase biases for both frequencies) were determined using the network platform and applied to about 300 single-receiver stations that act as dual-frequency PPP-RTK users. As numerically shown, over 120 minutes ( 2 hours) is required for $75 \%$ of the float errors to become less than $2 \mathrm{~cm}$, while $90 \%$ of which need more than 3 hours to attain $2 \mathrm{~cm}$. In case of the fixed errors however, after 30 minutes $75 \%$ of the errors become less than $2 \mathrm{~cm}$. After just 45 minutes, $90 \%$ of the ambiguity-fixed results become less than $2 \mathrm{~cm}$. A further outlook was provided of the improvements that one can expect when expanding the GPS-only case with the inclusion of BDS, Galileo and QZSS. All the results showed that integer ambiguity resolution provides for a very significant improvement of the convergence times, both for static and kinematic users, and that these times get significantly reduced further when more systems get integrated and/or ionospheric corrections become available. 


\section{Acknowledgements}

The development of Curtin's PPP-RTK Network Platform has been done in the context of the Positioning Program Project 1.19 'Multi-GNSS PPP-RTK Network' of the Cooperative Research Centre for Spatial Information (CRCSI) in Australia. The development of Curtin's Multi-GNSS PPP-RTK User Platform was done in the finalized CRCSI Positioning Program Project 1.01. The GNSS data were provided by Geoscience Australia. All this support is gratefully acknowledged. 


\section{References}

Australian Spatial Consortium (2012). Australian Strategic Plan for GNSS, available at http://www.crcsi.com.au/assets/Resources/4e4d9bcc-1173-4a22-92ed-e0d7f22fd326.pdf (consulted 10 June 2016).

Boehm J, Heinkelmann R, Schuh H (2007). Short note: a global model of pressure and temperature for geodetic applications. Journal of Geodesy, 81(10), 679-683.

CODE (2016). Center for Orbit Determination in Europe, http://aiuws.unibe.ch/ionosphere/p1c1.dcb (consulted 10 June 2016).

Collins P (2008) Isolating and estimating undifferenced GPS integer ambiguities. In: Proc ION NTM, pp 720-732

CRCSI (2016a). Cooperative Research Centre for Spatial Information, http://www.crcsi.com.au/research/1-positioning/114-development-of-analysis-centre-software/ (consulted 10 June 2016).

CRCSI (2016b). Cooperative Research Centre for Spatial Information, http://www.crcsi.com.au/research/1positioning/carrier-phase-positioning/ (consulted 10 June 2016).

CRCSI (2016c). Cooperative Research Centre for Spatial Information, http://www.crcsi.com.au/research/1-positioning/119-multi-gnss-ppp-rtk-network-processing/ (consulted 10 June 2016).

CRCSI (2016d). Cooperative Research Centre for Spatial Information, http://www.crcsi.com.au/research/1-positioning/121-ionospheric-modelling/ (consulted 10 June 2016).

De Jonge PJ (1998). A processing strategy for the application of the GPS in networks. PhD thesis, Delft University of Technology, the Netherlands. Publications on Geodesy 46, Netherlands Geodetic Commission.

Dow JM, Neilan RE, Rizos C (2009). The International GNSS Service in a changing landscape of Global Navigation Satellite Systems. Journal of Geodesy, 83(3), 191-198.

Elsobeiey M (2015). Precise Point Positioning using triple-frequency GPS measurements. Journal of Navigation, 68(3), 480-492.

Ge M, Gendt G, Rothacher M, Shi C, Liu J (2008) Resolution of GPS carrier-phase ambiguities in precise point positioning (PPP) with daily observations. J Geod 82(7):389-399

Guo F, Li X, Zhang X, Wang J (2016). Assessment of precise orbit and clock products for Galileo, BeiDou, and QZSS from IGS Multi-GNSS Experiment (MGEX). GPS Solutions, doi: 10.1007/s10291-016-0523-3.

ICD-GPS-200C (2000). Navstar GPS Space Segment / Navigation User Interfaces. Interface Control Document, Revision 12 April 2000.

IGS (2016). International GNSS Service, ftp://ftp.igs.org/pub/station/general/igs08.atx (consulted 10 June 2016).

IGS and RTCM-SC104 (2015). RINEX - the Receiver Independent Exchange Format, Version 3.03 , 14 July. International GNSS Service (IGS), RINEX Working Group and Radio Technical Commission for Maritime Services Special Committee.

Khodabandeh A, Teunissen PJG (2016). PPP-RTK and inter-system biases: the ISB look-up table as a means to support multi-system PPP-RTK. Journal of Geodesy, doi 10.1007/s00190-016-0914-9.

Kouba J, Heroux P (2001). Precise point positioning using IGS orbit products. GPS Solutions, 5(2), 12-28.

Laurichesse D, Mercier F (2007) Integer ambiguity resolution on undifferenced GPS phase measurements and its application to PPP. In: Proceedings of the 20th international technical meeting of the Satellite Division of The Institute of Navigation (ION GNSS 2007), pp 839-848

Lyard F, Lefevre F, Letellier T, Francis O (2006). Modelling the global ocean tides: modern insights from FES2004. Ocean Dynamics, 56(5), 394-415. 
Odijk D, Zhang B, Khodabandeh A, Odolinski R, Teunissen PJG (2016). On the estimability of parameters in undifferenced, uncombined GNSS network and PPP-RTK user models by means of S-system theory. Journal of Geodesy, 90(1), 15-44.

Petit G, Luzum B (2010). IERS Conventions 2010. IERS Technical Note No. 36, International Earth Rotation and Reference Systems Service, Central Bureau, BKG, Frankfurt am Main, Germany.

Saastamoinen J (1972). Atmospheric correction for the troposphere and stratosphere in radio ranging of satellites. In: Henriksen SW, Mancini A, Chovitz BH (eds). The use of artificial satellites in geodesy, Vol. 15, AGU Geophys. Monogr., Washington, 247-251.

Teunissen PJG (1990). An integrity and quality control procedure for use in multi sensor integration. In: Proceedings of ION GPS 1990, Colorado Springs, CO, 19-21 September, 513-522. Also published in: Volume VII of GPS Red Book: Integrated systems, ION Navigation, 2012

Teunissen PJG (1995). The least-squares ambiguity decorrelation adjustment: a method for fast GPS integer ambiguity estimation. Journal of Geodesy, 70(1), 65-82.

Teunissen PJG, Kleusberg A (1998). GPS for Geodesy. Springer-Verlag, Berlin-Heidelberg-New York.

Teunissen, PJG, Joosten P, Tiberius CCJM (1999). Geometry-free ambiguity success rates in case of partial fixing. Proceedings of ION NTM 1999, San Diego, CA, 25-27 January, 201-207.

Teunissen PJG, De Bakker, PF (2013). Single-receiver single-channel multi-frequency GNSS integrity: outliers, slips, and ionospheric disturbances. Journal of Geodesy, 87(2), 161-177.

Teunissen PJG, Khodabandeh A (2015). Review and principles of PPP-RTK methods. Journal of Geodesy, 89(3), $217-$ 240.

Wu J, Wu S, Hajj G, Bertiger W, Lichten S (1993). Effects of antenna orientation on GPS carrier phase measurements. Manuscripta Geodaetica, 18(2), 91-98.

Wübbena G, Schmitz M, Bagge A (2005). PPP-RTK: precise point positioning using state-space representation in RTK networks. In: Proceedings of ION GNSS 2005, Long Beach, CA, 13-16 September, 2584-2594.

Zumberge JF, Heflin MB, Jefferson DC, Watkins MM, Webb FH (1997). Precise point positioning for the efficient and robust analysis of GPS data from large networks. Journal of Geophysical Research, 102(3), 5005-5017. 
Table 1: Estimable dual-frequency network parameters, including their interpretation and conditions for existence.

\begin{tabular}{|l|c|c|}
\hline $\begin{array}{l}\text { Estimable } \\
\text { parameter }\end{array}$ & Notation \& interpretation & $\begin{array}{c}\text { Conditions } \\
\text { for existence }\end{array}$ \\
\hline Receiver ZTD & $\tau_{r}$ & $r \geq 1$ \\
\hline Receiver clock & $d \tilde{t}_{r}=\left(d t_{r}+d_{r, I F}\right)-\left(d t_{1}+d_{1, I F}\right)$ & $r \geq 2$ \\
\hline Satellite clock & $d \tilde{t}^{s}=\left(d t^{s}+d_{I F}^{s}\right)-\left(d t_{1}+d_{1, I F}\right)$ & $s \geq 1$ \\
\hline Receiver phase bias & $\tilde{\delta}_{r, j}=\left(\delta_{r, j}-\frac{1}{\lambda_{j}}\left[d_{r, I F}-\mu_{j} d_{r, G F}\right]+z_{r, j}^{1}\right)-\left(\delta_{1, j}-\frac{1}{\lambda_{j}}\left[d_{1, I F}-\mu_{j} d_{1, G F}\right]+z_{1, j}^{1}\right)$ & $j \geq 1 ; r \geq 2$ \\
\hline Satellite phase bias & $\tilde{\delta}_{, j}^{s}=\left(\delta_{, j}^{s}-\frac{1}{\lambda_{j}}\left[d_{, I F}^{s}-\mu_{j} d_{, G F}^{s}\right]\right)-\left(\delta_{1, j}-\frac{1}{\lambda_{j}}\left[d_{1, I F}-\mu_{j} d_{1, G F}\right]\right)-z_{1, j}^{s}$ & $j \geq 1 ; s \geq 1$ \\
\hline Ionospheric delay & $\tilde{\iota}_{r}^{s}=\iota_{r}^{s}+d_{r, G F}-d_{, G F}^{s}$ & $r \geq 1 ; s \geq 1$ \\
\hline Phase ambiguity & $\tilde{z}_{r, j}^{S}=\left(z_{r, j}^{s}-z_{r, j}^{1}\right)-\left(z_{1, j}^{s}-z_{1, j}^{1}\right)$ & $j \geq 1 ;$ \\
& $d t_{1}, d_{r, 1}, d_{r, 2}, d_{, 1}^{s}, d_{, 2}^{s}, \delta_{1, j}, z_{r, j}^{1}, z_{1, j}^{s}$ & $r \geq 2 ; s \geq 2$ \\
\hline $\begin{array}{l}\text { S-basis (non- } \\
\text { estimable parameters) }\end{array}$ & $j \geq 1 ;$ \\
\hline
\end{tabular}

Note: $(\cdot)_{, I F}=\frac{\mu_{2}}{\mu_{2}-\mu_{1}}(\cdot)_{, 1}-\frac{\mu_{1}}{\mu_{2}-\mu_{1}}(\cdot)_{, 2} ;(\cdot)_{, G F}=-\frac{1}{\mu_{2}-\mu_{1}}\left[(\cdot)_{, 1}-(\cdot)_{, 2}\right]$

Table 2: Estimable dual-frequency ionosphere-corrected PPP-RTK user parameters, plus their interpretation.

\begin{tabular}{|l|c|c|}
\hline $\begin{array}{l}\text { Estimable user } \\
\text { parameter }\end{array}$ & Notation \& interpretation & $\begin{array}{l}\text { Conditions } \\
\text { for } \\
\text { existence }\end{array}$ \\
\hline Receiver position & $x_{u}$ & \\
\hline Receiver ZTD & $\tau_{u}$ & \\
\hline Receiver clock & $d \tilde{t}_{u}=\left(d t_{u}+d_{u, I F}\right)-\left(d t_{1}+d_{1, I F}\right)$ & $j \geq 1$ \\
\hline Receiver phase bias & $\tilde{\delta}_{u, j}=\left(\delta_{u, j}-\frac{1}{\lambda_{j}}\left[d_{u, I F}-\mu_{j} d_{n e t, G F}\right]+z_{u, j}^{1}\right)-\left(\delta_{1, j}-\frac{1}{\lambda_{j}}\left[d_{1, I F}-\mu_{j} d_{1, G F}\right]+z_{1, j}^{1}\right)$ & $j \geq 1$ \\
\hline Receiver code bias & $\tilde{d}_{u, j}=\mu_{j}\left(d_{u, G F}-d_{n e t, G F}\right)$ & $j \geq 1 ; s \geq 2$ \\
\hline Phase ambiguity & $\tilde{z}_{u, j}^{s}=\left(z_{u, j}^{s}-z_{u, j}^{1}\right)-\left(z_{1, j}^{s}-z_{1, j}^{1}\right)$ & $j \geq 1$ \\
\hline $\begin{array}{l}\text { S-basis (non- } \\
\text { estimable } \\
\text { parameters) }\end{array}$ & $d_{u, 1}, d_{u, 2}, z_{u, j}^{1}$ & \\
\hline
\end{tabular}

Table 3: Estimable single-frequency ionosphere-corrected PPP-RTK user parameters, plus their interpretation.

\begin{tabular}{|l|c|c|}
\hline $\begin{array}{l}\text { Estimable user } \\
\text { parameter }\end{array}$ & Notation \& interpretation & $\begin{array}{l}\text { Condition } \\
\text { for } \\
\text { existence }\end{array}$ \\
\hline Receiver position & $x_{u}$ & \\
\hline Receiver ZTD & $\tau_{u}$ & \\
\hline Receiver clock & $d \tilde{t}_{u}=\left(d t_{u}+d_{u, 1}-\mu_{1} d_{n e t, G F}\right)-\left(d t_{1}+d_{1,1}-\mu_{1} d_{1, G F}\right)$ \\
\hline Receiver phase bias & $\tilde{\delta}_{u, 1}=\left(\delta_{u, 1}-\frac{1}{\lambda_{1}}\left[d_{u, 1}-2 \mu_{1} d_{n e t, G F}\right]+z_{u, 1}^{1}\right)-\left(\delta_{1,1}-\frac{1}{\lambda_{1}}\left[d_{1,1}-2 \mu_{1} d_{1, G F}\right]+z_{1,1}^{1}\right)$ & \\
\hline Phase ambiguity & $\tilde{z}_{u, 1}^{s}=\left(z_{u, 1}^{s}-z_{u, 1}^{1}\right)-\left(z_{1,1}^{s}-z_{1,1}^{1}\right)$ & $s \geq 2$ \\
\hline $\begin{array}{l}\text { S-basis (non- } \\
\text { estimable } \\
\text { parameters) }\end{array}$ & $d_{u, 1}, z_{u, 1}^{1}$ & \\
\hline
\end{tabular}


Table 4: Overview of a-priori corrections for PPP-RTK network and user processing.

\begin{tabular}{|l|l|l|}
\hline Correction & Magnitude & Source \\
\hline Antenna phase centre offsets & $\begin{array}{l}0.5-3 \mathrm{~m} \text { (satellite); } \\
5-15 \mathrm{~cm} \text { (receiver) }\end{array}$ & IGS ANTEX file (IGS, 2016) \\
\hline Antenna phase centre variations & $\begin{array}{l}5-15 \mathrm{~mm} \text { (satellite); } \\
<3 \mathrm{~cm} \text { (receiver) }\end{array}$ & IGS ANTEX file (IGS, 2016) \\
\hline Tidal (solar earth, polar) effects & $<40 \mathrm{~cm}$ & IERS Conventions 2010 models (Petit and Luzum, 2010) \\
\hline Ocean loading & $1-10 \mathrm{~cm}$ & FES2004 global ocean tide model (Lyard et al., 2006) \\
\hline P1-C1 differential code biases ${ }^{1}$ & $<5 \mathrm{~m}$ & IGS/CODE P1-C1 DCB file (CODE, 2016) \\
\hline Phase windup ${ }^{2}$ & $10 \mathrm{~cm}$ & Correction according to Wu et al. (1993) \\
\hline Relativistic effects & $10-20 \mathrm{~m}$ & Periodic relativity correction (ICD-GPS-200C, 2000) \\
\hline Tropospheric delays & $2-25 \mathrm{~m}$ & $\begin{array}{l}\text { Standard troposphere model, e.g. Saastamoinen's model } \\
\text { (Saastamoinen, 1972) or GPT model (Boehm et al., 2007) }\end{array}$ \\
\hline
\end{tabular}

${ }^{1}$ only applied to code data; ${ }^{2}$ only applied to phase data

Table 5: Additional estimable code bias parameters in a multi-frequency $(f \geq 3)$ case, including their interpretation.

\begin{tabular}{|l|c|c|}
\hline $\begin{array}{l}\text { Estimable } \\
\text { parameter }\end{array}$ & Notation \& interpretation & $\begin{array}{l}\text { Conditions } \\
\text { for existence }\end{array}$ \\
\hline Receiver code bias & $\tilde{d}_{r, j}=\left(d_{r, j}-\left[d_{r, I F}+\mu_{j} d_{r, G F}\right]\right)-\left(d_{1, j}-\left[d_{1, I F}+\mu_{j} d_{1, G F}\right]\right)$ & $j \geq 3 ; r \geq 2$ \\
\hline Satellite code bias & $\tilde{d}_{, j}^{s}=\left(d_{, j}^{s}-\left[d_{, I F}^{s}+\mu_{j} d_{, G F}^{s}\right]\right)-\left(d_{1, j}-\left[d_{1, I F}+\mu_{j} d_{1, G F}\right]\right)$ & $j \geq 3 ; s \geq 1$ \\
\hline
\end{tabular}

Table 6: Frequencies of the GNSS constellations used for multi-GNSS PPP-RTK of NNOR.

\begin{tabular}{|l|l|}
\hline GNSS constellation & Frequencies used \\
\hline GPS & L1 L2 \\
\hline BDS (BeiDou) & B1 B2 \\
\hline Galileo & E1 E5a E5b \\
\hline QZSS & L1 L2 L5 \\
\hline
\end{tabular}




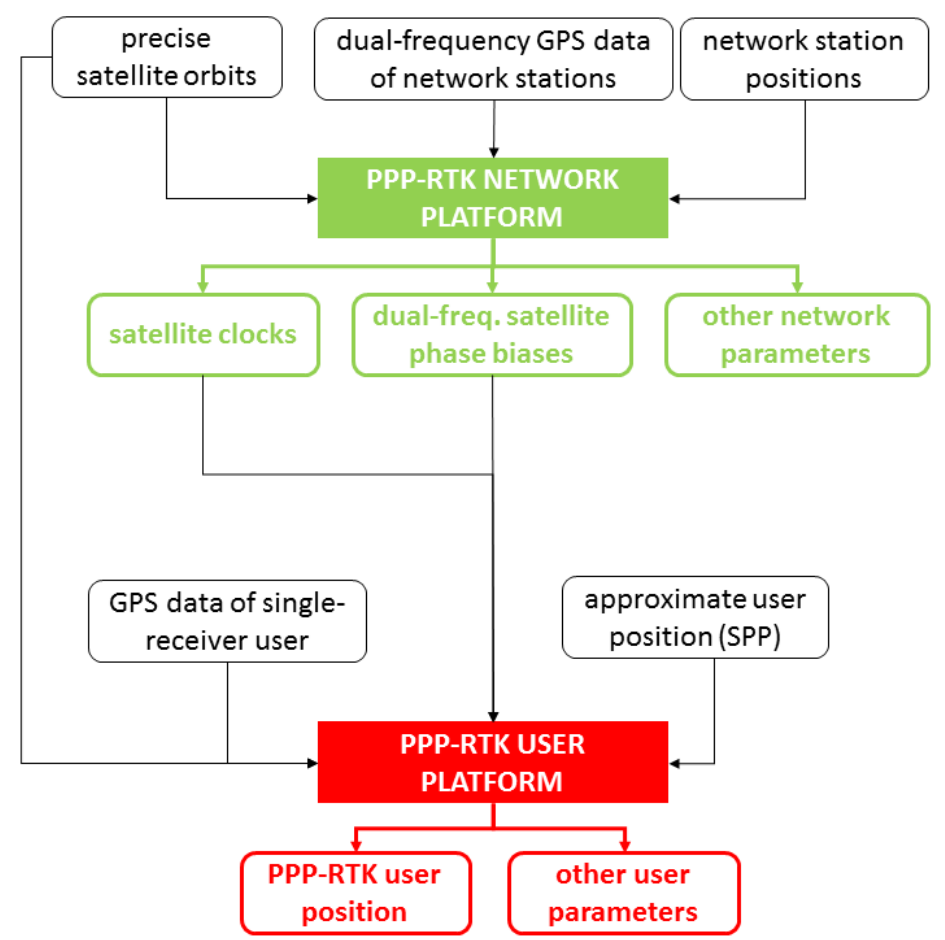

Figure 1: Curtin's PPP-RTK Platform consists of a Network component and User component. 


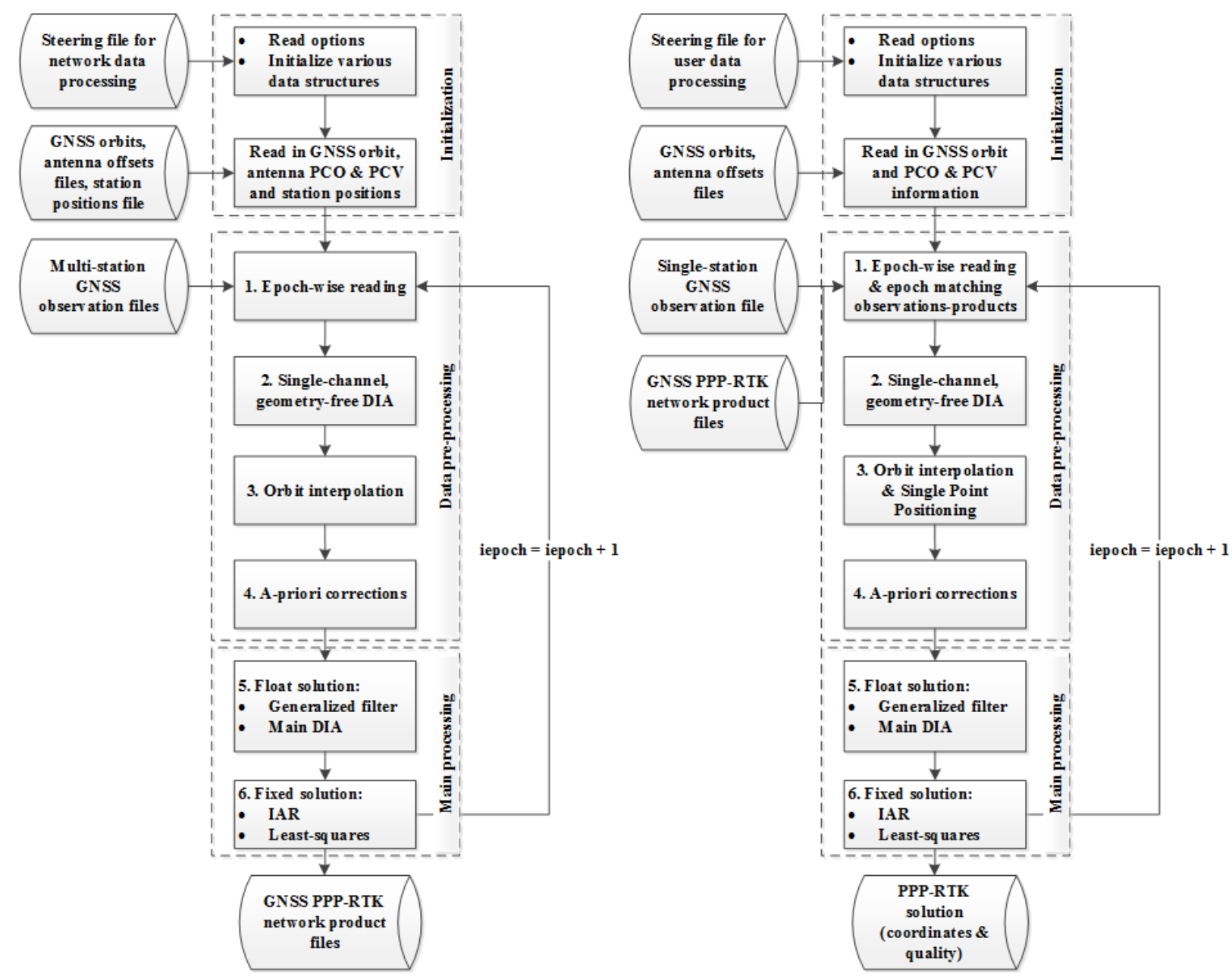

Figure 2: Flowcharts of Curtin's PPP-RTK Network Platform software (left) and Curtin's PPP-RTK User Platform software (right). 


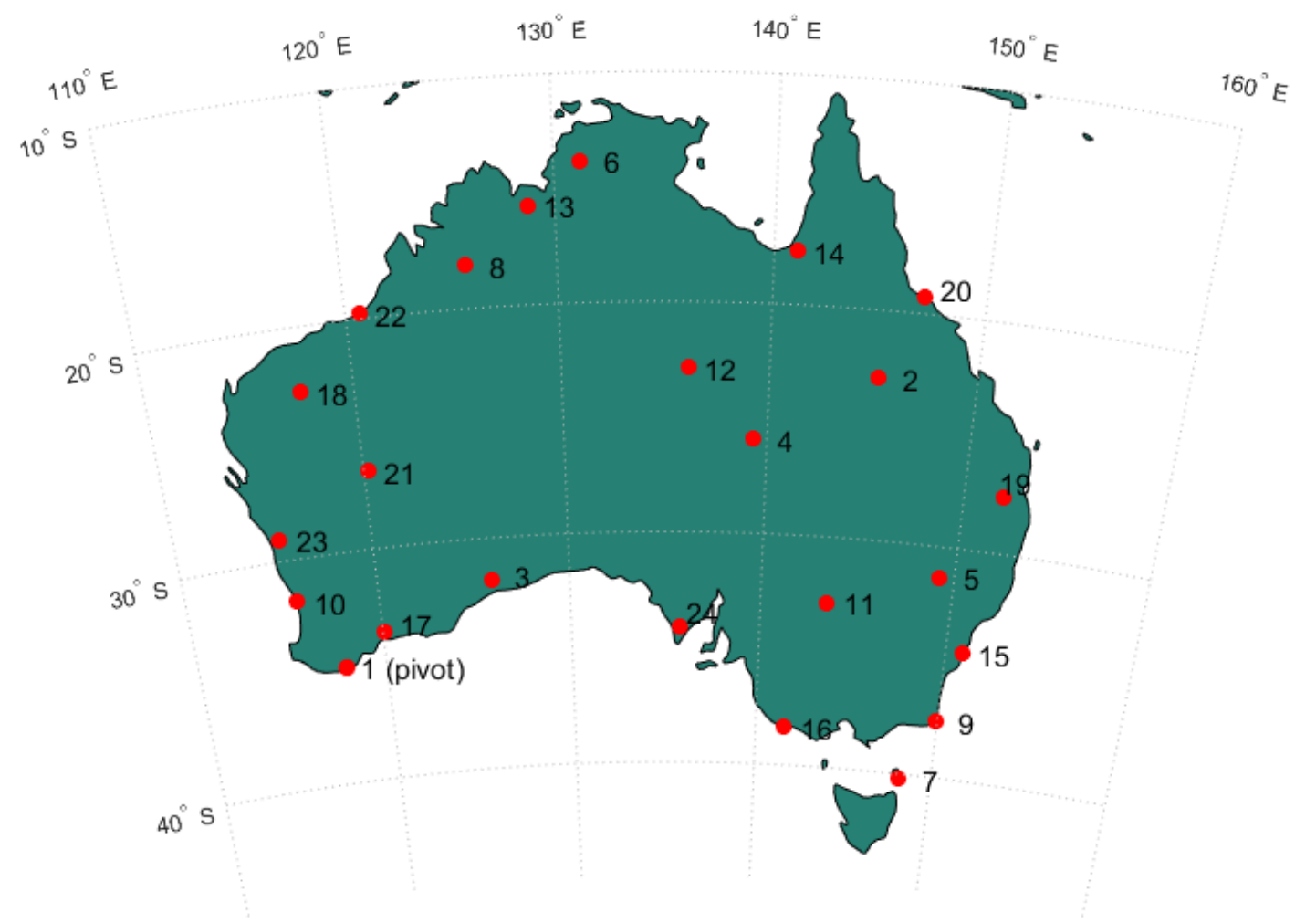

Figure 3: Geographic distribution of the Australian GPS CORS stations used for the PPP-RTK network processing (red circles).

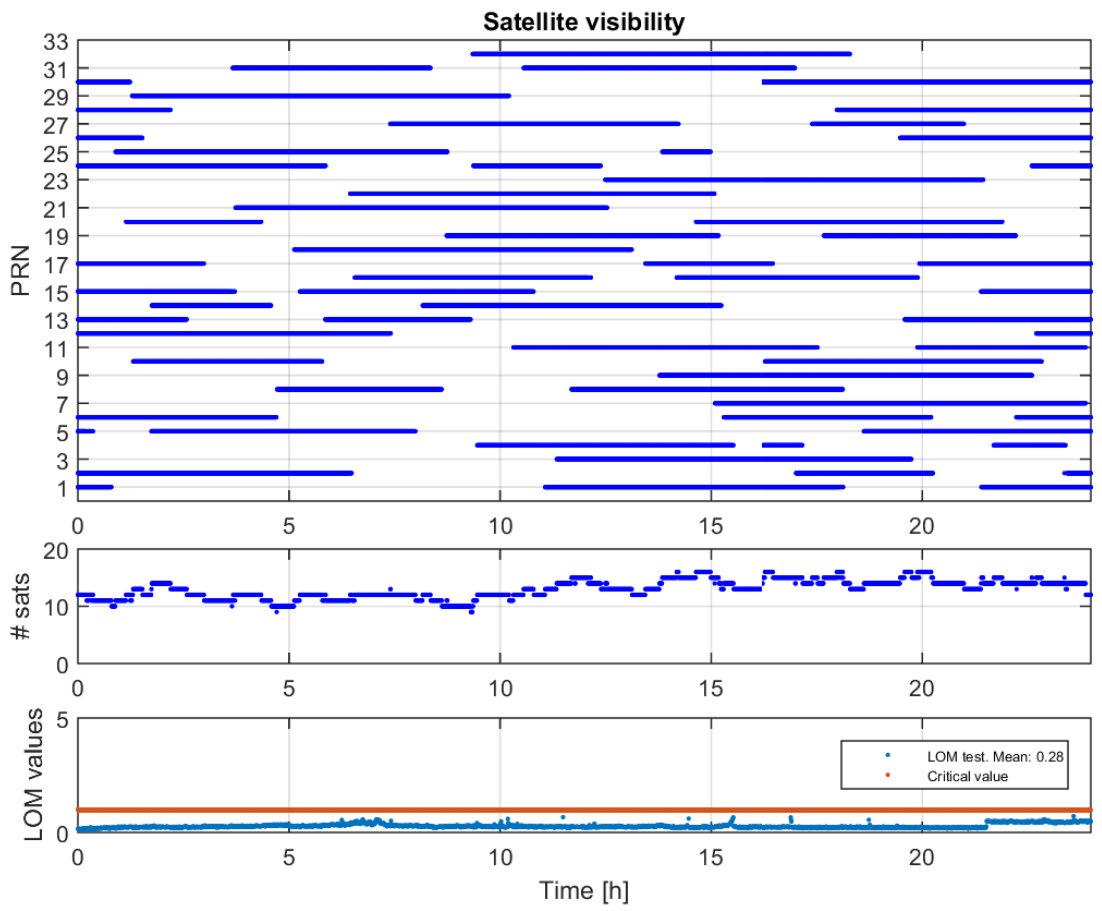

Figure 4: Satellite visibility, number of satellites and LOM test outcomes during the day. 

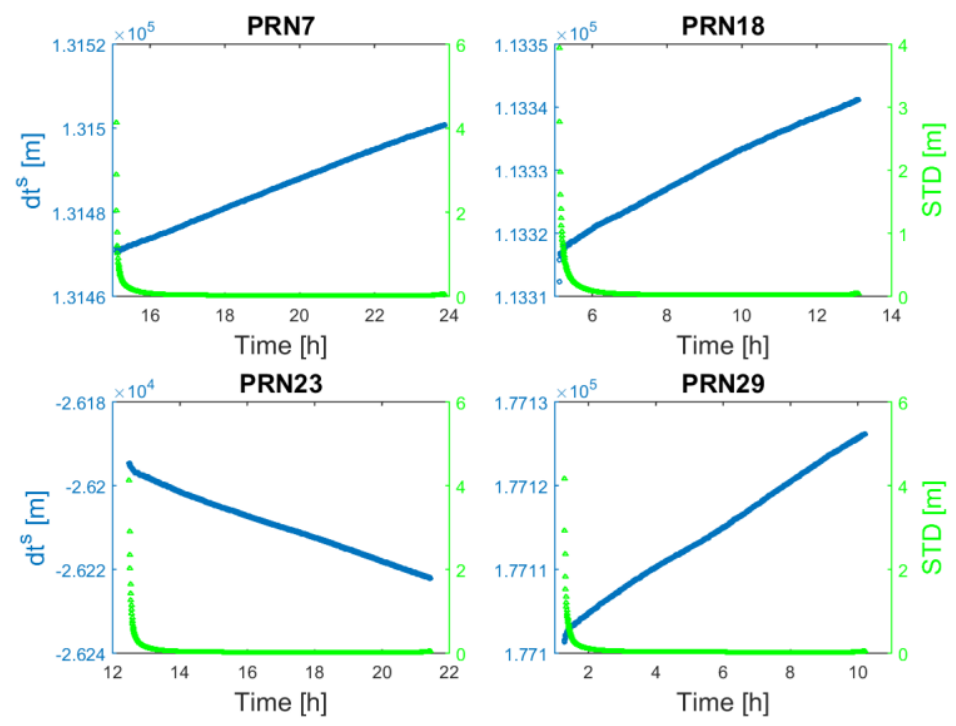

Figure 5: Satellite clock estimates along with their formal standard deviations (STD) for 4 representative satellites.
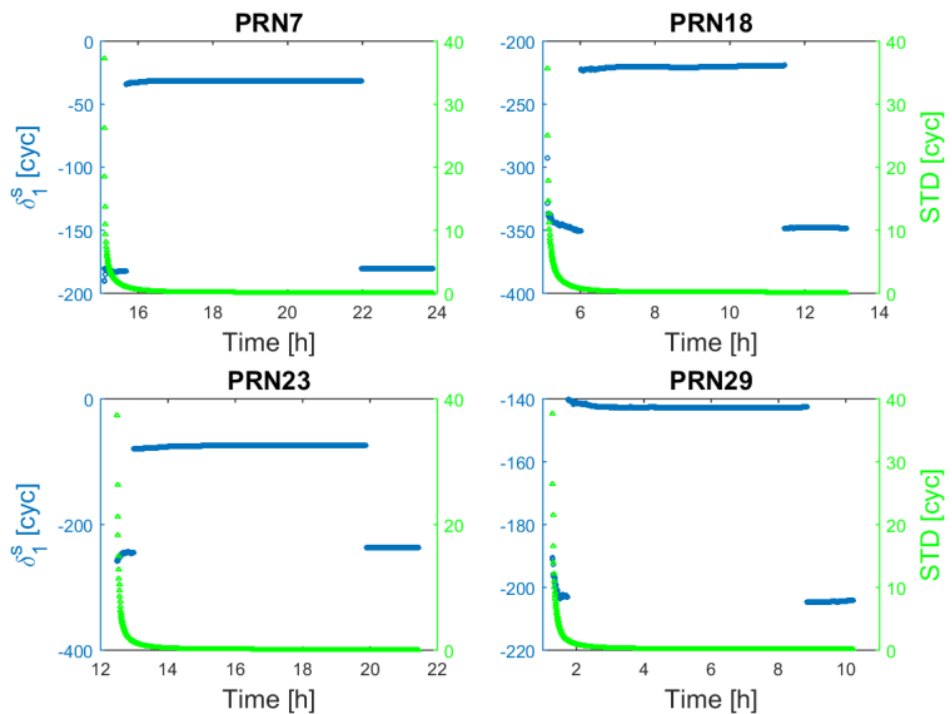

Figure 6: Satellite phase bias estimates for L1 [cycle] along with their formal standard deviations (STD) for 4 representative satellites. 

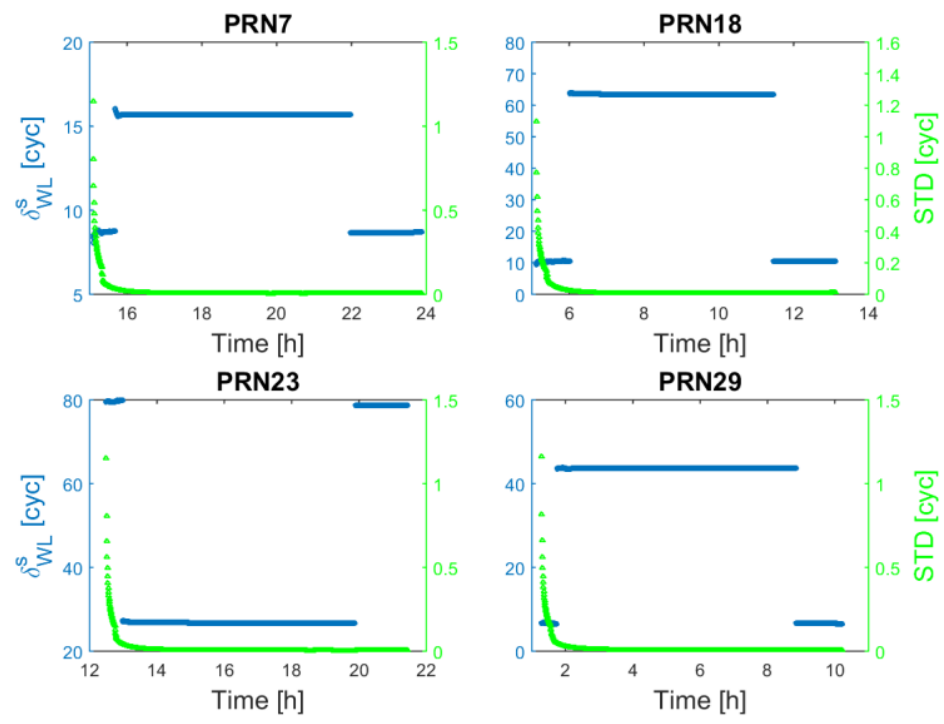

Figure 7: Satellite phase bias estimates for wide-lane [cycle] along with their formal standard deviations (STD) for 4 representative satellites.
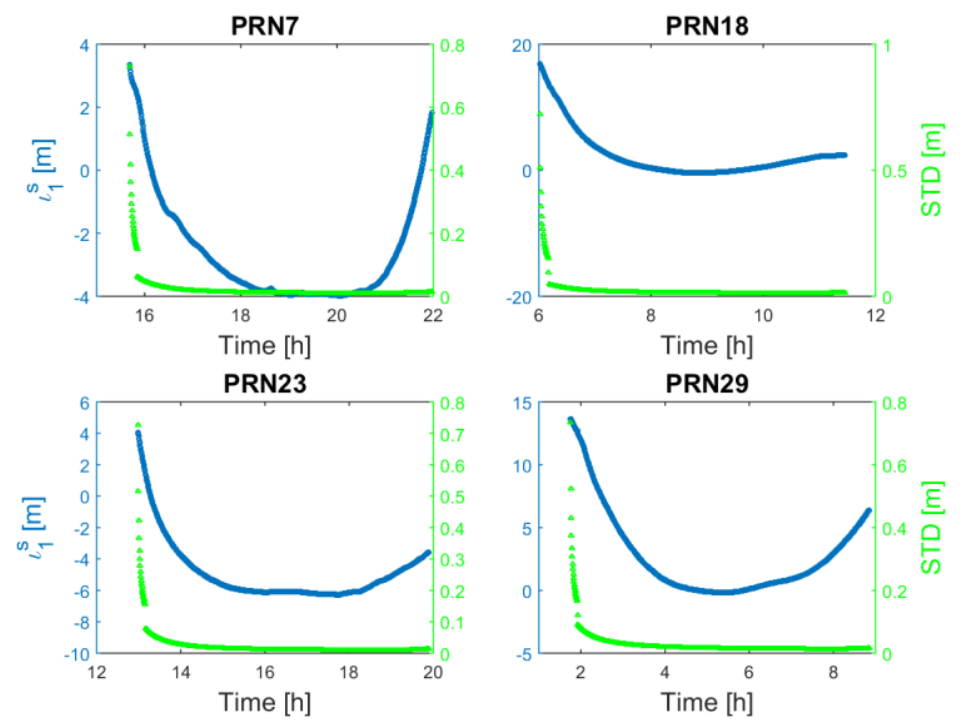

Figure 8: Slant ionospheric delay estimates, along with their formal standard deviations (STD), for 4 representative satellites. 

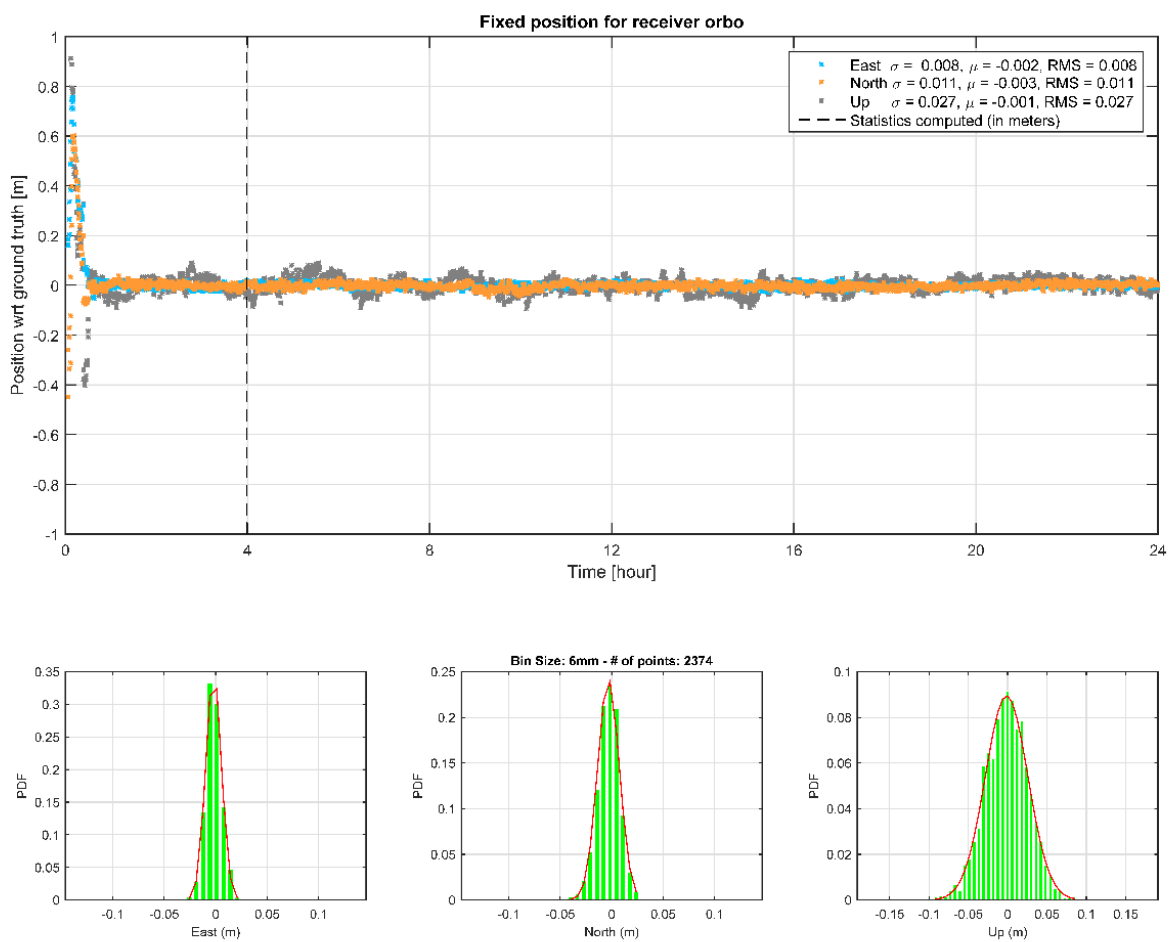

Figure 9: Time-series and histograms of the ambiguity-fixed PPP-RTK kinematic user position (in East-North-Up) for station ORBO, relative to its ground-truth. The standard deviations and RMS values are calculated for the positions after 4 hours (vertical line).
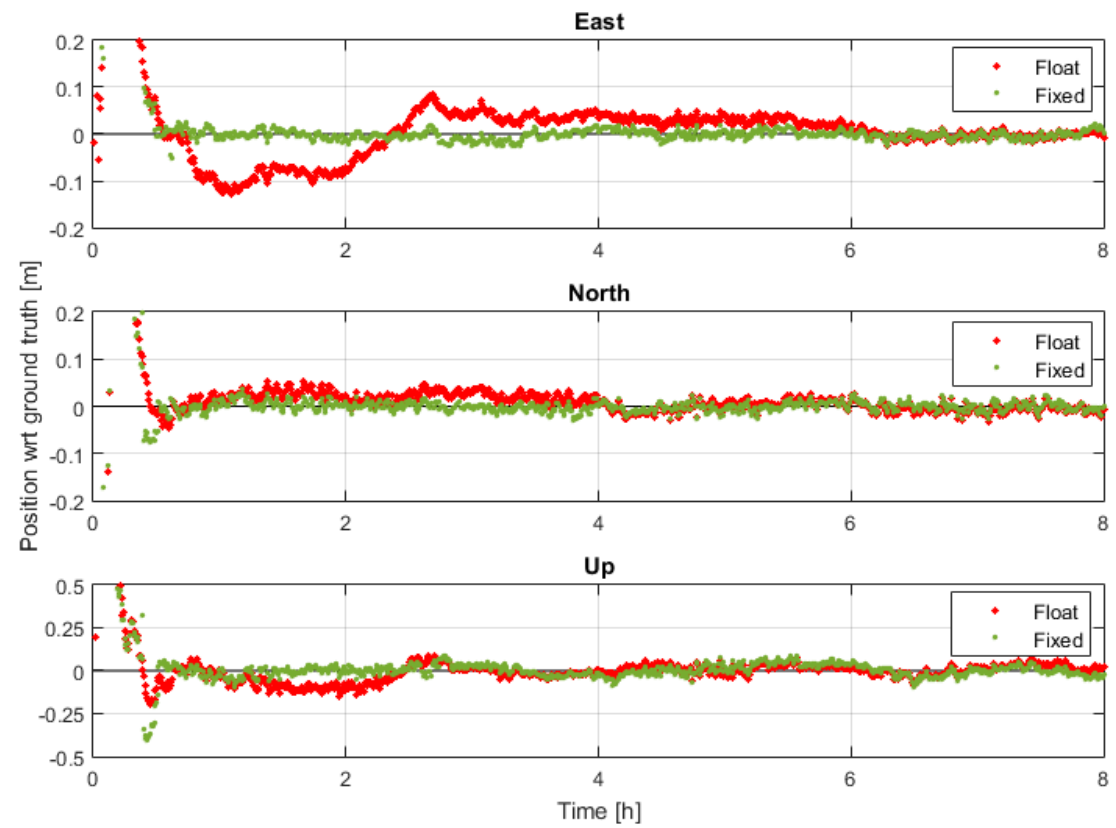

Figure 10: Time-series (8 hours) of the PPP-RTK kinematic user position (in East-North-Up) for station ORBO: ambiguity-float (red dots) and ambiguity-fixed (green dots). 

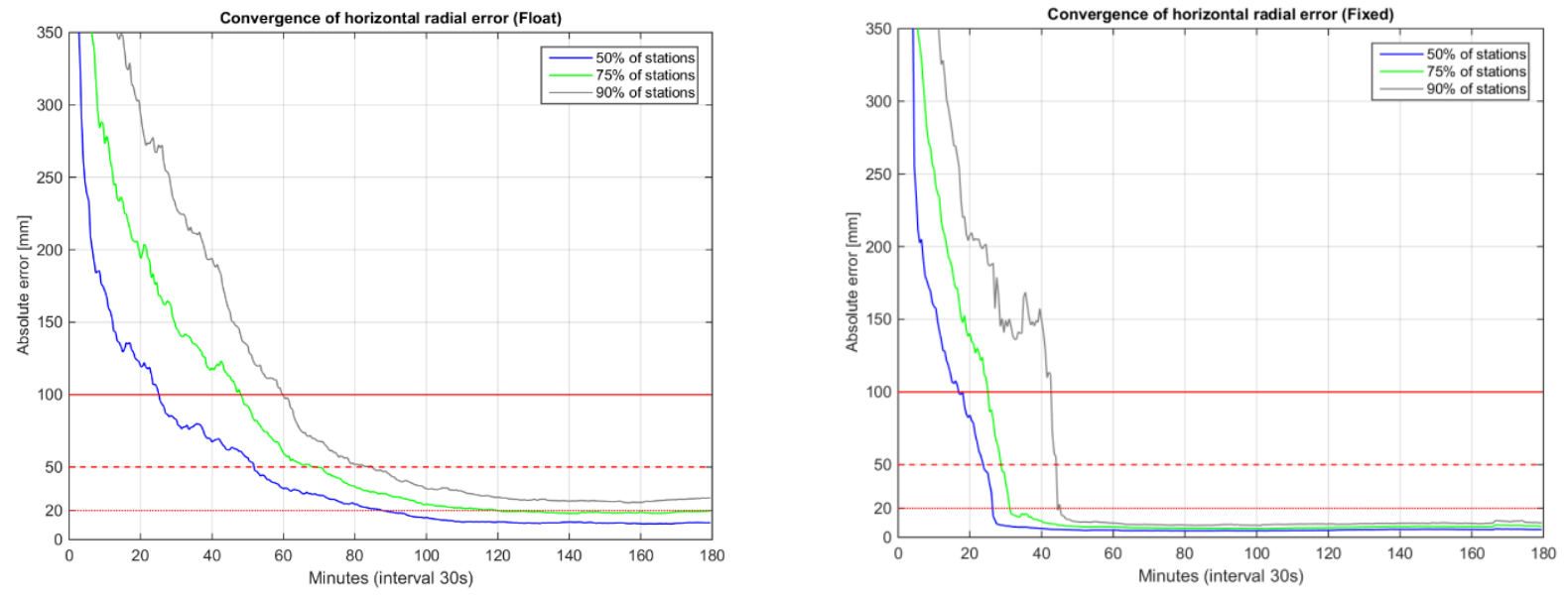

Figure 11: Convergence behaviour of the horizontal radial position errors on the basis of about 300 PPP-RTK user stations with 30 seconds sampling interval that are processed in static mode. Left: ambiguity-float; right: ambiguity-fixed.
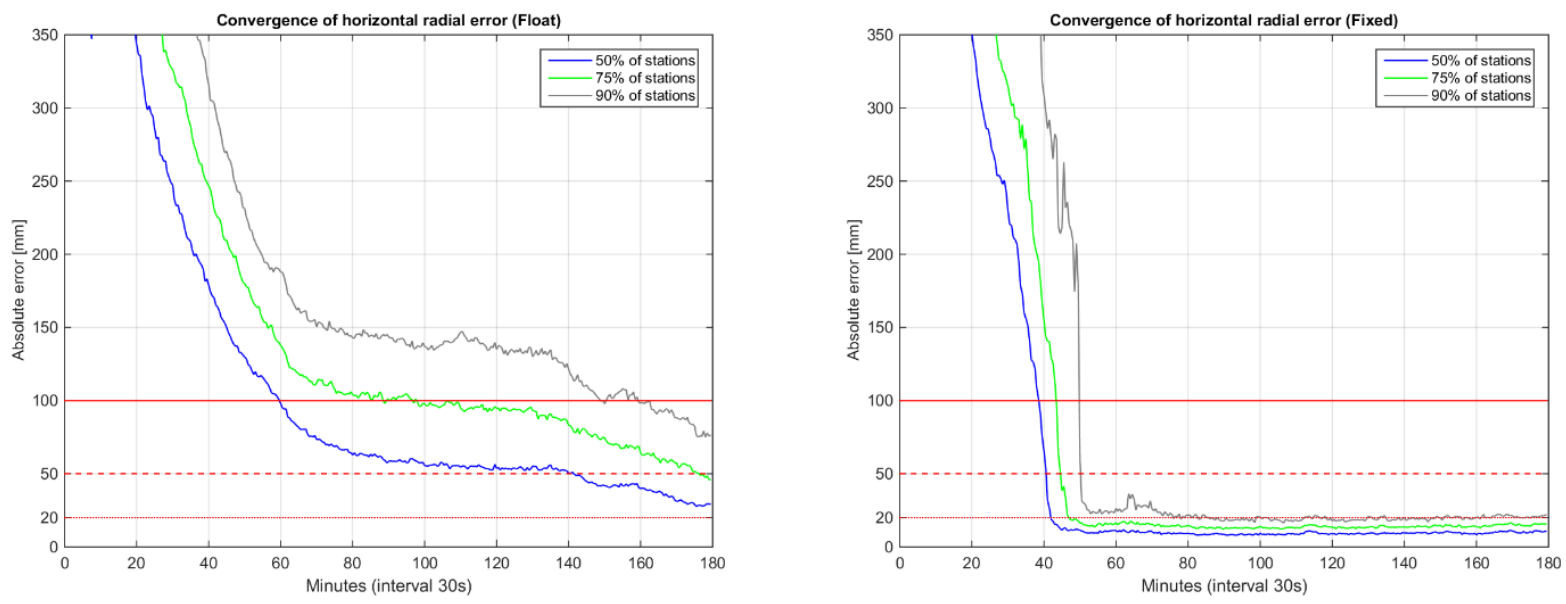

Figure 12: Convergence behaviour of the horizontal radial position errors on the basis of about 300 PPP-RTK user stations with 30 seconds sampling interval that are processed in kinematic mode. Left: ambiguity-float; right: ambiguity-fixed. 
PPP-RTK user fixed convergence time [min]

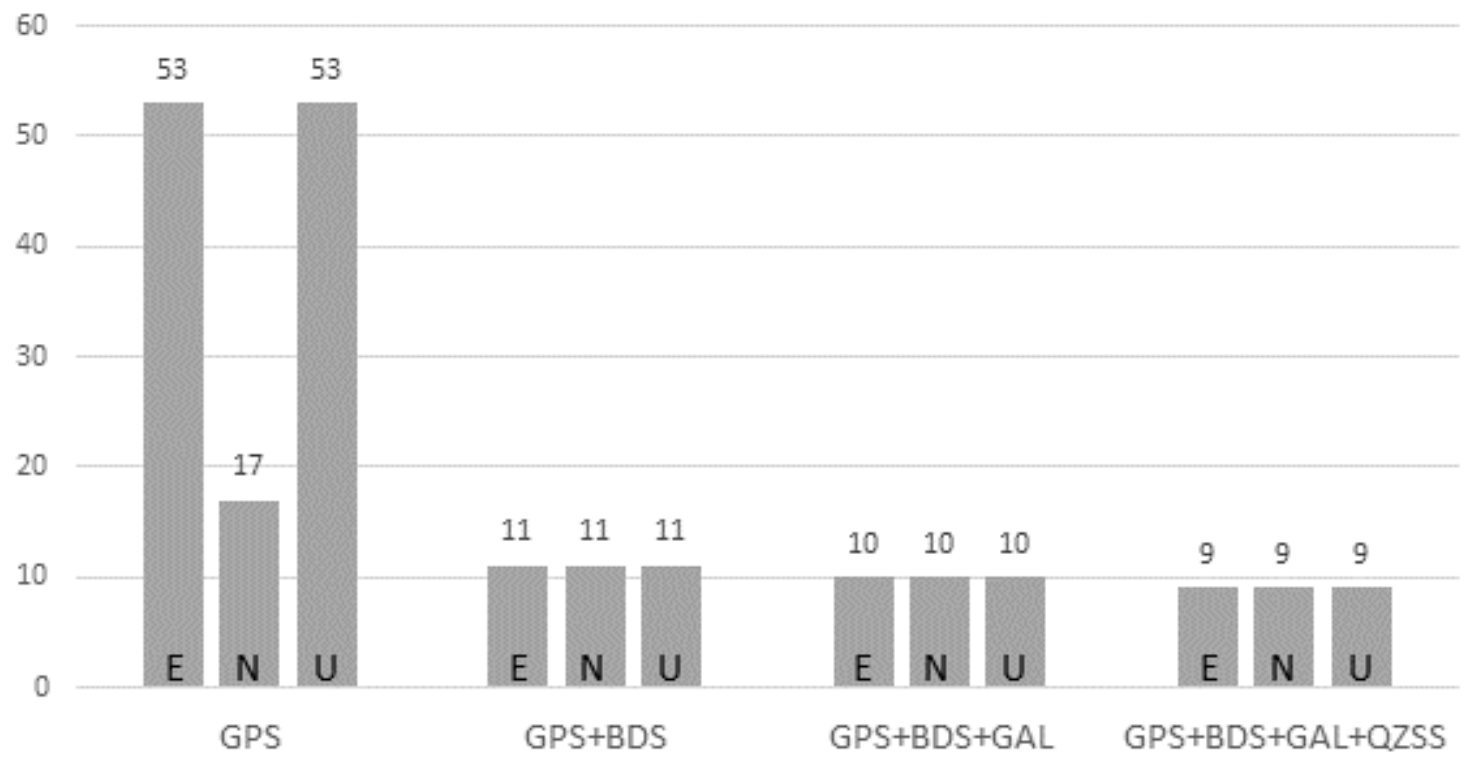

Figure 13: Convergence times corresponding to East (E) and North (N) positioning errors of $5 \mathrm{~cm}$ and Up (U) positioning error of $10 \mathrm{~cm}$ for several multi-GNSS PPP-RTK scenarios for user WLAL, in case the ambiguities are fixed.
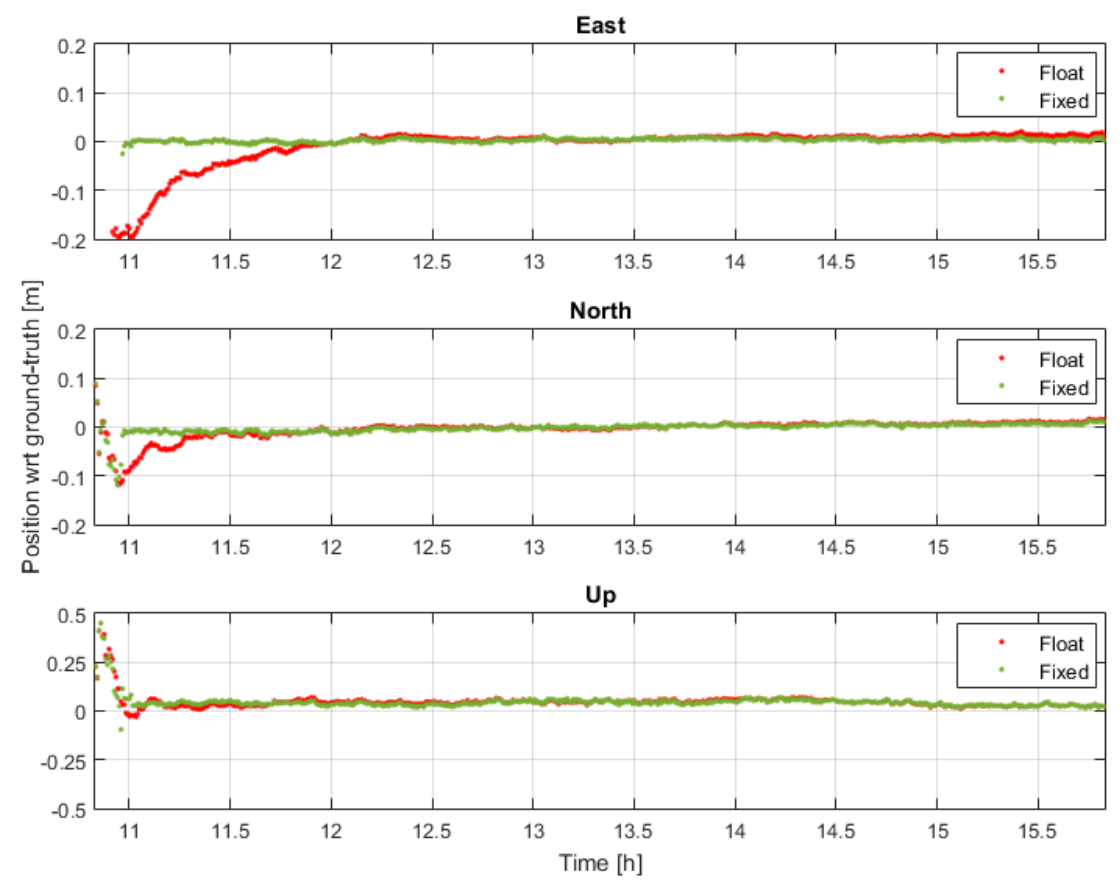

Figure 14: Time-series (5 hours) of the PPP-RTK kinematic user position (in East-North-Up) for station WLAL, estimated using GPS, $B D S$, Galileo and QZSS: ambiguity-float (red dots) and ambiguity-fixed (green dots). 